\title{
3-Way Handshake PAKE Based on Reversible Cellular Automata
}

Sofiane Baghor

Computer science department-UDL University

Algeria

baghorsoufiane@yahoo.fr

Kamel Mohamed Faraoun

Computer science department, UDL University

Sidi bel abbes

Algeria

kamel_mh@yahoo.fr

\begin{abstract}
In this paper, we propose only three way handshake password authenticated key exchange using only the mechanism of two-dimensional reversible cellular automata. The scheme use simple and elementary operations to handle the problem of mutual authentication between two communicating parties, and establish common secure, valid session key based only on a short, easily memorable password. Security of the proposed scheme shown to be high, while it resist most common attacks on authentications schemes and provide the perfect forward secrecy. Besides, runtime performances of the authentication protocol are very competitive, and outperform those of existing similar schemes. The proposed schemes ensure the three authentication requirements, namely the mutuality, the authenticity and the key exchange using only elementary and parallelizable operations.
\end{abstract}

Keywords: Password Authentication, Mutual Authentication, Key Exchange, Two-Dimensional Reversible Cellular Automata

Received: 8 January 2018, Revised 12 March 2018, Accepted 18 March 2018

(C) 2018 DLINE. All Rights Reserved

\section{Introduction}

Entity authentication and secure key exchange are two important building blocks of any multi-user secure distributed/centralized system. While the former aims to prove the identity of a given user and allow it to gain access to a given service, the later aims at establishing a cryptographic key that enable secure communication between the user and the requested provider during a predetermined communication's session. The user owns a specific and personal information (that can be either a password or a smart card) that enables his authentication within a server, and permits to establish a valid cryptographic key in a secure manner. An eavesdropper or a man in the middle should not be able to obtain enough information permitting to brute force or guess the password, and any unauthorized user should be able to impersonate the server and take the identity of legitimate user.

Combining both entity authentication and secure key exchange leads to the definition of a Password Authenticated Key Exchange (PAKE) protocols permitting to achieve a secure communications between two or more parties based only on the knowledge of single password.

\begin{tabular}{llllll}
\hline 50 & Journal of Information Security Research & Volume & 9 & Number 2 & June \\
\hline
\end{tabular}


A solution to the key exchange problem first introduced by Diffie-Hellman [1] using exponential algorithm. However, this scheme neither provides authentication nor resists against man-in the-middle attack. It is necessary to verify the identities of the communicating parties, when they establish a connection. This authentication is usually provided in combination with a key exchange protocol between the parties.

The concept of PAKE was first introduced by Bellovin and Merritt in 1992 [2] and there after many PAKEprotocols have been proposed and analyzed [3-8]. Most of these schemes involves modular exponentiations with high computational overhead and communication bandwidth, which is unsuitable for low power applications.

In this paper, we propose an only three way handshake password authenticated key exchange using only the mechanism of twodimensional reversible cellular automata. Thus, the scheme provides a high security and performances level with an optimized computational cost. The proposed scheme handle remote entity authentication, session key exchange and mutual authentication between two communicating parties using a simple and elegant computational model, and it is shown to be resistant against common related attacks.

The remaining of the paper is organized as follows: Section 2 presents related works on password authenticated key exchange using cellular automata, and Section 3 gives elementary details on the cellular automata mechanism. In section 4, the proposed authentication scheme is fully described and detailed, while related security analysis is presented in Section 5 and comparisons analysis are presented in Section 6. Finally, conclusions are drawn in section 7.

\section{Related Works}

Recently, some password-based authentication protocols have been implemented by cellular automata since it has been accepted as a good calculation model and have been used in various domains but little used in authentication systems.

D. K. Bhattacharryya \& S. Nandimade the first attempt [9] who proposed an authentication protocol that allows two parties that share a small password to generate a common cryptographic key using simple cellular automata operations to simplify the logical structure. However, this scheme uses numerous instances CA-based hash function, is not resistant to the stolen session key attack, is not robust against the attack on known key security, hence the protocol does not provide perfect forward secrecy. In addition, the protocol can be executed only by the presence of a shared secret key.

In [10] S Tripathy \& S Nandi (2006) proposed a password-based authentication protocol using bi-dimensional reversible cellular automata. This protocol provides mutual authentication and key agreement on an insecure channel, it is also robust against all passive and active attacks while providing perfect forward secrecy, but through the addition of more instances of hash function, that increases the computational overhead of the scheme.

Finally, authentication based on the A Secure CA Based 2-Party Key Exchange Protocol was proposed in [11] using the group and non-group characteristics of cellular automata. The security of the protocol is based primarily on asymmetric encryption, where each participant has a certified key pair. The protocol depends on a PKI infrastructure and a complete management of keys (certification request, verification of the validity of certificates, etc.).Another required module is the generator of the secret from the digital fingerprint; this requires complicated processes and expensive devices for companies. In addition to these limitations, we note an application limit in the case of an application (user-to-user authentication); the prior sharing of the digital fingerprint remains a problem.

\section{Cellular Automata Preliminaries}

A cellular automaton consists of a number of cells arranged in a regular lattice, when each cell has its own state changing in a discrete time step. States of the CA's cells are updated synchronously using a local transition rule defining each new cell's state using its old state, and the states of the corresponding neighbors. The neighbors are a specific selection of cells relatively chosen with respect to a given cell's position that can be defined for each cell using a radius $r$ on the lattice. This gives $2 r+1$ different neighbor including the cell itself.

The boundaries cells of the lattice are concatenated together in a cyclic form to deal with finite size automaton. If the same update rule is used for all the cells then the resulting CA is named uniform. Otherwise, if a different transition rule is used each 
time the cell's position change, the resulting $C A$ is named non-uniform.

Formally, a $C A$ is defined by 4-tuple $(d, S, N$, $f)$ where,

$\bullet d$ dimension $1,2,3 \ldots$

- $S$ : finite state set $\{1,2,, \ldots ., n\}$

- $N$ : Neighborhood vector $\left(x_{1}, . . x_{m}\right) \in Z^{m}$

- $f$ : Local update rule mapping from $S^{m}$ to $S$.

If we define the state of a cell $i$ at a time $t$ with $s_{i}^{t}$, its state on the time $t+1$ depends only on the states of the corresponding neighborhood at time $t$, by applying a transition rule that define the way states are updated. If the neighborhood radius is $r$, and if two cell states are defined ( 0 or 1 ), then the length of each transition rule is then $2^{2 \mathrm{r}+1}$ bit, and the number of possible rules is equal to $2^{\wedge}\left(2^{2 r+1}\right)$.

This feature of simple local-rule leading to complex global-behavior motivated many researchers to design security primitives. Interested reader is referred to [12]-[16].

The transition rule of one dimensional binary CAs is generally coded using the integer value of the corresponding binary representation, when the different CA's configurations are represented by binary blocks.

Given some evolution rule, a sequence of states has a single evolution, but it can be generated by one, many or no ancestors. A cellular automaton is reversible if each configuration has a single ancestor. Such a cellular automaton is called reversible cellular automata and is briefly explained in next.

\subsection{Reversible Cellular Automata (RCA)}

Reversible Cellular Automata (RCA) are dynamical systems, which conserve their initial information. For this reason, they present a very interesting mathematical theory, and have been used as models for data ciphering [19],information coding [18] and simulation of reversible physical phenomena [17] among other applications. The theory of Reversible Cellular automata (RCA), are realized in [20-22]. Algorithms for finding reversible one-dimensional CA are proposed in [19].

CA A is reversible (called invertible) if its global map $G_{A}$ is invertible i.e. there exists another automaton $B$ (withglobal map $G_{B}$ ) such that $G_{B}=G_{A}{ }^{-1}$. Here the automaton $B$ is called the inverse of $A$. For every possible configuration $C$ : $G_{B} 0 G_{A}(C)=C$. Therefore, for every possible configuration the global mapspecifies one and only one successor. However, the problem of deciding, whether a configuration has apredecessor is proved to be NLOG complete for one-dimensionaland NP-complete for all higher $(d \geq 2)$ dimensional CA [23].

Basing on this theory, RCA can be used as crypto system. Let $T=(d, S, N, f)$ be an RCA and $T^{-1}=\left(d, S, N^{\prime}, f^{\prime}\right)$ be its inverse. The message $M$ is written in $(d \geq 2)$ dimensional array using symbols in $S$. Now the cipher can be obtained by applying $T$ on $M$. In reverse, operating $T^{-1}$ on the cipher, the original message can be deduced. Higher dimensional $(d \geq 2)$ RCA is to be used for this purpose, as all one-dimensional RCA can be effectively inverted [24]. Apart from that, the RCA to be used in the proposed system is required to have the following properties.

- Given the RCA $T$ it is computationally infeasible to find $T^{-1}$;

- However, it is easier to find $T$ by knowing $T^{-1}$.

Composition of some easy invertible RCA can be used for this purpose. Selecting some easily invertible RCA $\left(T_{1}, T_{2} \ldots T_{n}\right)$, and their composition $T=\left(T_{1} \mathrm{o} T_{2} \ldots \mathrm{o} T_{n}\right)$ can be published and used as a public key keeping $T_{1}, T_{2} \ldots T_{n}$ insecret. $T^{-1}=\left(T_{n}^{-1} \mathrm{o} T_{n-1}{ }^{-1} \ldots \mathrm{o}\right.$ $T_{1}^{-1}$ ) can be used as private key without sacrificing the secrecy. The proposed scheme exploits such RCA, which is illustrated in the next section.

$52 \quad$ Journal of Information Security Research Volume 9 Number 2 June 2018 


\section{The Proposed Scheme}

\subsection{The Issue}

The problem comes down to the ability to overcome a brute force attack to find the secret (the low entropy password). Therefore, to design a strong password-based authentication system, we need to consider the following particular properties and requirements:

- Users have only a low entropy secret. More precisely, it is possible for the opponent to search in all possible secrets within a reasonable time.

- Offline dictionary attacks should not be possible. This means that a passive indiscretion that registers the transcription of one or more sessions cannot eliminate a large number of possible passwords.

- Online dictionary attacks should not be possible. This means that the active opponent cannot abuse the protocol in order to eliminate a large number of possible passwords. An active opponent can always eliminate at least one password per attempt to pass himself off as the legitimate user by using this password.

Our main contribution is the minimization of the number of message flows exchanged during the authentication and key confirmation phase, as well as the minimization of expensive computational operations such as (hash functions ...), ensuring a high level of security.

\subsection{Protocol Design Assumptions}

Hypothesis 1: This is a case where the opponent can listen to all messages sent or received. This is a realistic assumption in typical communication systems.

Hypothesis 2: Another realistic hypothesis where the opponent can intercept, modify and replay messages.

Hypothesis 3: Again, a hypothesis very realistic - it is widely believed that internal participants are often more a threat that foreigners. This brings us to another security hypothesis.

Hypothesis 4: A typical scenario is where two participants commit to a shared key protocol and one returns a challenge that was intended for itself. This attack can only be possible if parallel execution of the same protocol is allowed, but this is often a realistic assumption.

\subsection{Protocol Architecture}

There are three characteristics that we consider architectural criteria to classify different authentication protocols and cryptographic key establishment: The existing cryptographic keys, how the session key is established and the number of proto colusers is designed to serve.

\subsubsection{Existing Cryptographic Keys}

It is not possible to establish an authenticated session key without existing secure channels already available. Therefore, if two participants want to establish a new session key, there are essentially three options:

- The principals already share a secret;

- The existing of an offline certificate authority server in which it verifies the authenticity and the validity of a certified public keys;

- The presence of an online server (trusted third party) where all participants trust. In order to pass information between participants it may be necessary to pass it via the on-line server.

We opted for the first option (the principals already share a secret), since our protocol is based only on a shared easy-toremember password. The major advantage of this choice is that it allows our protocol to be used in a decentralized system architecture and direct user-to-user applications. 
Architectural Design 1: In our protocol, Alice and Bob share a pre-shared secret (an easy-to-remember password) to ensure the authentication of the entity and the establishment of a secure session key.

\subsubsection{How the Session Key is Established?}

There are three ways to generate session keys in a key establishment protocol: the first one is a key transport mechanism in which some participants assign a valid session key and that key will be sent to other participants. The second: this is a key agreement mechanism in which the session key is contribution-based by all protocol participants. While the third is a hybrid method of first two ways.

In our architecture we used the key transport method; where one of the participants generates a valid session key and transport it securely to the other party. It allowed us to significantly reduce the number of messages exchanged during the authentication phase and the key confirmation phase.

Architectural Design 2: In our protocol, the session key is generated by Bob and transported to Alice safely (Key Transport Protocol).

\subsubsection{Number of Users}

A final component of the protocol architecture is the number of principals that take part in the protocol. Not to be confused with the maximum number of users supported by the protocol. Here we talk about how many users contribute at a time to the key establishment phase. Most key establishment protocols (including ours) have concentrated on the case where two users wish to establish a session key for point-to-point communications. To extend these to conference key protocols can complicate matters a great deal. So, these protocols are not addressed among the goals of our approach.

In our protocol only the participant concerned contributes to the phase of generation of the session key; and then transport it to the peer entity. Therefore, only legitimate parties will be aware of the session key after running the protocol.

Architectural Design 3: In our scheme, only Bob contributes to generate the session key and transport it to the peer entity (Alice).

\subsection{The Desired Goals}

We will cite in this section a detailed list of desired goals by our protocol, the list is illustrated in the following table:

The protocol must ensure authentication of the entity; i.e. one of the parties must be assured of the identity of the second party involved in the protocol and that second party must have actually participated;

The protocol must provide mutual authentication between Bob and Alice; That is to say, the first goal must be ensured in both directions;

The protocol must establish a fresh session key, known only to session participants;

The protocol must provide key integrity;

The protocol must provide key confirmation from Alice to Bob i.e. That Alice must have the assurance that the session key $K_{i}$ is a good key to communicate with Bob, and that Bob is in possession of that key.

The purpose of the protocol is to provide mutual trust in the $K i$ key, i.e., Bob's prediction that $K_{s}$ is a good key for use with Alice, and Alice wishes to communicate with Bob using the Ki key, which Alice believes is good for this use.

Finally, the protocol aims to provide forward secrecy i.e. if the long-term secret is compromised, session keys that have been previously established using this secret should not be compromised too.

Table 1. The desired goals

\subsection{What do we want to protect? Against what dangers?}

The confidentiality of the session key and the shared secret is mandatory in order to counteract the passive attacks. In addition 
to passive attacks: it is necessary for designers of authentication protocols to propose techniques in order to counteract active attacks.

The following table presents the set of attacks that we wish to counteract during the design and construction of our protocol. In order to clarify the objectives of our protocol. Since for a protocol to achieve particular objectives, it depends on its strength to thwart all attacks considered possible.

\begin{tabular}{|l|}
\hline Password guessing attack (Online/Offline); \\
\hline Replay attack and clock un-synchronization; \\
\hline Mutual authentication and man in the middle attack; \\
\hline Session key agreement, forgery attack and known key security; \\
\hline No verifier-table stored and stolen verifier attack; \\
\hline Reflection attack; \\
\hline Denial Of Service (DOS). \\
\hline
\end{tabular}

Table 2. List of possible attacks

\subsection{Protocol Construction}

This approach is a theoretical design that depends on any cryptographic mechanism (symmetric, asymmetric and one-way function). This is called modularity of the crypto-system. We let total freedom for the integrator of our solution to choose any cryptographic mechanism if it is safe and proven, this choice is made as required.

We recommend mechanisms based on proven cellular automata in order to obtain a fast, light and efficient implementation. For symmetric cryptography, we opt for the crypto-system of our proven approach [22];for asymmetric cryptography we recommend the crypto-system of Kari [23] and for the one-way functions we choose [26] from [24-26].

Using the mechanism of second-order reversible cellular automata, we propose a new system PAKE Password Authenticated Key Exchange. The security of our approach relies solely on a small low entropy password (PWi) shared between the two communicators (Alice and Bob), and on the difficulty of reversing a two-dimensional RCA Reversible Cellular Automata.

The concept of challenge in a similar way to the principle of nonces. We use the previous session key Ki-1 as a challenge instead of generating and sending new nonces in each execution, which reduces the number of messages exchanged during the execution of the protocol. The use of this challenge at the same time eliminates the time synchronization problem between the participants.

The $\mathrm{Ki}-1$ key is used in a challenge-response scenario in which both participants retain the value of the previous one-time session key of each session. Alice then sends $K i-1$ encoded by the derived S key from the shared password ( $P W i)$ in order to meet the challenge Bob is expecting. Alice checks the validity of the challenge, otherwise Bob refuses Alice's request.

The proposed authentication system comprises two phases: an authentication and key exchange phase, and a phase of confirmation of key knowledge. The following notations are adopted in the remainder of this article:

Our protocol dispense stage registration since the protocol needs only a small password to be completed successfully. This phase allows mutual authentication between the two parties (Alice and Bob), so that each verifies the identity of the other. After the authentication phase, a shared session key is set up to enable secure communication between the two parties during a predetermined communication session. An intruder who has access to the messages exchanged should not be able to draw information about the secret S, or the established session key Ki.

\subsubsection{Authentication and Key Exchange Phase}

The phase of mutual authentication and key exchange takes place in 3 steps: 


\begin{tabular}{|l|l|}
\hline$H():$ & A one-way cryptographic function \\
\hline$\psi():$ & The expansion function \\
\hline$K_{i-1}:$ & The previous session key used as a challenge \\
\hline$I D_{i}:$ & The identifier of the user Ui (Alice) \\
\hline$P W_{i}:$ & The user's password Ui (Alice) \\
\hline$S:$ & The secret key derived from the $P W i$ \\
\hline$K_{i}:$ & The current session key \\
\hline$T:$ & The public key generated by Alice \\
\hline$T^{1}:$ & The generated private key corresponding to $T$, generated by Alice \\
\hline
\end{tabular}

Table 3. The rating table

- MAKE-1: For Alice who wishes to authenticate with Bob, he must first enter his identifier IDi and his password PWi to start an access session:

- A secret key $\mathrm{S}$ is derived from the password $P W i$;

- Alice generates a reversible cellular automaton $\mathrm{T}$ and its inverse $T^{-1}$;

- Then, Alice calculates the cryptogram $X=E_{s}\left(K_{i-1}, T\right)$;

- Finally, the couple $<I D_{A}, X>$ is sent to Bob.

Note that Alice does not need to wait for a challenge from Bob because he is aware of the challenge $\left(K_{i-1}\right)$ he needs to code it and send it to Bob. Bob on his side waits for him to receive the challenge in the form of a cryptogram so that he can authenticate Alice.

- MAKE-2: When receiving the above pair, first Bob must retrieve the key $K_{i-1}$ from the cryptogram $X=E s\left(K_{i-1}, T\right)$ using the secret key $S$ so that, It can authenticate Alice. After comparing the recovered key to the key of the previous stored session, Bob authenticates and accepts Alice's request in case of a tie, otherwise the user's request is denied.

In order to perform mutual authentication, Bob must also prove its identity to Alice. To do this, the received ephemeral public key is used. Bob generates a communication key for the current session and then encodes this key and the previous session key under the cryptogram $Y=T(K i-1, K i)$ using the ephemeral public key $T$. Alice tries to recover the encoded challenge New fresh and unique shape. If the equality is verified, then Bob is authenticated with Alice. If not, Bob is considered an intruder, and the connection is interrupted. Figure 1 illustrates the fully detailed diagram.

\begin{tabular}{|c|c|c|}
\hline $\begin{array}{l}\text { Alice (Initiator) } \\
\mathrm{S}\end{array}$ & & $\begin{array}{c}\text { Bob (Responder) } \\
\mathrm{S}\end{array}$ \\
\hline \multicolumn{3}{|c|}{ Generate RCA T and $\mathrm{T}^{-1}$} \\
\hline \multirow[t]{2}{*}{$\mathrm{X}=\mathrm{E}_{\mathrm{s}}\left(\mathrm{K}_{\mathrm{i}-1}, \mathrm{~T}\right)$} & $I D_{A_{4}} \mathrm{X}$ & \\
\hline & $\mathrm{Y}, I D_{B}$ & $\begin{array}{l}E_{s}(X) \Rightarrow T, K_{i-1} \\
\text { Verifies } K_{i-1} \\
\text { generate } K_{i} \\
Y=T\left(K_{i}, K_{i-1}\right)\end{array}$ \\
\hline $\begin{array}{l}T^{-1}(\mathrm{Y}) \Rightarrow \mathrm{K}_{\mathrm{i}}, \mathrm{K}_{\mathrm{i}-1} \\
\text { Verifies } \mathrm{K}_{\mathrm{i}-1}\end{array}$ & & \\
\hline$H\left(K_{i}\right)$ & $\mathrm{H}\left(\mathrm{K}_{\mathrm{i}}\right), I D_{A}$ & $\left.H\left(K_{i}\right)\right)=?$ \\
\hline
\end{tabular}

Figure 1. Proposed Authenticated Key Exchange Diagram 


\subsubsection{Key Confirmation Phase}

After mutual authentication has been successfully completed, both parties must have the knowledge of the key used for secure communication in the current session confirmed. The session key $K_{i}$ is generated by Bob and sent to Alice during the messages exchanged during the authentication phase (MAKE-2). A spy that collects all the exchanged messages is unable to deduce all the information about the session key since Alice is the only one who owns the $T^{-1}$ decryption private key.

- KC-1:The confirmation of Bob's key (Ki) by Alice is an integral part of the message (MAKE-2) since the key transport mechanism is used, because one of the characteristics of cryptographic algorithms is the Inability to amend part of the encrypted message. The verification of the previous session key implies the integrity of the new session key.

- KC-2: Confirmation of the Alice key with Bob is done by sending the currently hashed session key $H(K i)$; it is clear that this message is fresh and unique and cannot be forged by a malicious user. On receipt of this hash, Bob performs the same operation and compares the two hashes; if they are equal, Bob will be sure that Alice has the right communication key. Finally, a secure communication can be carried out.

\section{Experimental Results}

In the following, we discuss the safety properties of the proposed scheme in relation to the potential joint attacks. It is assumed that the attacker has the potential to listen to the communication channel between Alice and Bob and can modify any message exchanged. A comparative table is also presented in the following section between the proposed scheme and some existing CAbased authentication schemes.

\subsection{Password Guessing Attack}

In the proposed scheme, the user's password $P W i$ is transformed into a secret key $S$ by means of an expansion function $\psi$ where $S=\psi(P W i)$. In addition, the secret key derived from the password is used to encrypt the RCA T (which is itself generated randomly) and the previous session key $K_{i-1}$, knowing that $T$ and $K_{i-1}$ have not been transmitted anywhere in the clear on the network. The only password packet passed from Alice to Bob contains $\left\langle I D, X=E_{S}\left(K_{i-1}, T\right)>\right.$. As a result, the attacker is unable to retrieve information about the password from the identifiers and messages being played between Alice and Bob, so there is no way to mount an offline attack to guess the password (off-line password guessing attack).

Finally, to resist an online attack (on-line password guessing attack), the system limits the number of attempts to a limited number of times.

\subsection{Replay Attack and Clock un-synchronization}

The time stamp-based authentication system may suffer from the replay attack since the transmission delay is unpredictable in a network environment. For this reason we use a nonce based model for the proposed scheme instead of a timestamp-based design, so that the system can prevent such a serious problem from the non-synchronization of the clock. In order to better optimize our system the nonces are not generated every time to launch a challenge, but the idea is to use the previous session key $K_{i-1}$ as a nonce that is both known by Alice and Bob.

During the authentication phase, an opponent can claim to be a valid user and identify himself to the server (Bob) by sending messages that were previously transmitted by a legitimate user (Alice). During the authentication phase, an opponent can claim to be a valid user and attempts to connect to the server by sending him messages previously transmitted by a legitimate user. To avoid such an attack, the user generates an unpredictable and different RCA T for each session, and the server generates an novel fresh session key $\mathrm{Ki}$ which makes messages exchanged from a given session valid only for that session, and could not be reused in other sessions. Therefore, an opponent who replays a previous $X$ message is unable to perform the last phase of the protocol.

Therefore, an opponent cannot overcome the verification process with the server with legitimate values previously collected.

Obviously, the replay attack fails. Therefore, the proposed scheme can withstand a replay attack.

\subsection{Mutual Authentication and Man in the MiddleAttack}

It is a basic requirement of an authentication system that the user and server authenticate themselves to each other. In the 
proposed scheme, the server authenticates the user by verifying the authentication message using any symmetric encryption mechanism. If the server successfully retrieves $K_{i-1}$ from the connection request message, the server confirms the legitimacy of the user. The server then responds with a specific message $\left(Y=\left(T\left(K_{i}, K_{i-1}\right)\right)\right.$ to the user, which contains the new session key with the old one in order.The user authenticate the server by checking this pair, which cannot be constructed only by the legitimate server.

Therefore, mutual authentication is guaranteed by the MAKE-1 and MAKE-2 steps of the protocol. We know that the user's spoof attack and the fake server attack cannot be invoked by a malicious user $U_{z}$. Therefore, it is impossible for $U_{z}$ to conduct a man-in-the-middle attack. It is obvious that the protocol is resistant to man in the middle attack while ensuring mutual authentication.

\subsection{Session Key Agreement, forgery Attack and known Key Security}

After a successful mutual authentication, a session key is safely transported, and finally shared between the client and the server for use during the rest of the communication session. Our protocol ensures that the session key is known only by the legitimate entities (the client and the server). Since Alice generates the RCA $T$ and its inverse $T^{1}$ randomly and freshly, so only Alice can retrieve the current session key from the $Y$ cryptogram; therefore, no third party can send a forged message to the client or server. Since the challenge $\left(K_{i-1}\right)$ and the randomly generated $R C A$ are different for each session, even if a given session key is compromised, neither the secret key nor the session keys (past and future) will be compromised. Therefore, the proposed scheme ensures (known key security).

\subsection{No Verifier-table Stored and Stolen Verifier Attack}

As long as neither Alice nor Bob store no verification table, authentication depends only on a small, low-entropy password. As a result, the system resists storage table modification attack and ensures lower storage cost. Bob uses the secret key $S$ computed from the shared password PWi to authenticate Alice while guaranteeing the freshness of the cryptogram; While Alice uses the $R C A T$ and its inverse $T^{-1}$ generated during the authentication phase to authenticate Bob. Therefore, the proposed system is robust against stolen verifier attack.

\subsection{Reflection Attack}

A reflection attack can only be performed if and only if parallel executions of the same protocol are allowed. For example, if a participant is an Internet host, it can accept sessions from several other participants. In our case, a reflex attack cannot be performed even if the protocol allows several parallel executions for two reasons. The first reason - no challenge runs in clear during the authentication phase. While de second - even if a challenge can be unveiled or predicted, such an attack cannot be accomplished because of the Alice's way to authenticate Bob (By an asymmetrical mechanism)which is completely different to theBob's way to authenticate Alice (by asymmetric mechanism).

\subsection{Perfect Forward Secrecy}

The idea of forward secrecy is that when a long-term key (S) is compromised, session keys that were previously established with this long-term key should not be compromised too. The crucial concept for providing forward secrecy is the principle of the ephemeral public key (in our case we used the RCA $T$ and its inverse $T^{-1}$ ); This is a public key $T$ which is used only during the duration of the key establishment protocol and is then destroyed with its corresponding private key $T^{1}$. If the public key is used only to authenticate the session key, and the session key cannot be retrieved without the ephemeral private key, then the key $K_{i}$ cannot be retrieved from the cryptogram $Y=T\left(K_{i}, K_{i-1}\right)$ without knowledge of the private key $T^{1}$. Therefore, our protocol provides perfect forward secrecy.

\section{Comparative Analysis}

In order to illustrate the advantages of the proposed system, a comparative study was carried out against several existing CAbased authentication schemes. It is clear that the proposed approach ensures all the listed properties while requiring less computational cost.

Furthermore, our proposed approach is superior to other studies in terms of security aspects and operating performance.

\subsection{Security Analysis}

The comparison is made with respect to the security. Table 4 presents several aspects of security provided by the proposed scheme compared to most known CA-based authentication schemes proposed in [9], [10] and [11]. 


\subsubsection{The Limitations and Weaknesses of CPAKE [9]}

This protocol is not resistant to stolen session key attack; if the attacker obtains the session key $K_{i}$ by chance, then the secret key m can be determined using the information eavesdropped $\left(Q_{A}, Q_{B}\right)$ during phase 1 , this cancompletely break the protocol. However, this scheme depends only on the security of the hash function, since the password p is not needed for the calculation of the session key. Therefore this protocol does not resist neither against offline password guessing attack nor against stolen verifier attack.

The disclosure of $m$ is sufficient to reveal the antecedent and subsequent conversations. Because the intermediate secrets $\left(Q_{A}\right.$, $Q_{B}$ ) for each session are sent in clear on the network. Therefore, this approach does not ensure neither perfect forward secrecy propriety nor known key security propriety.

\begin{tabular}{|l|l|l|l|l|}
\hline Security Criterion & CPAKE[9] & RPAKE[10] & CA-KEP[11] & Proposed \\
\hline Perfect forward secrecy & No & Yes & Yes & Yes \\
\hline known key security & No & Yes & Yes & Yes \\
\hline Stolen session key attack & No & Yes & Yes & Yes \\
\hline Offline-password guessing & No & Yes & Yes & Yes \\
\hline Resistance to Stolen verifier attack & No & Yes & No & Yes \\
\hline Resistanceto replay attack & Yes & Yes & Yes & Yes \\
\hline Mutualauthentication & Yes & Yes & Yes & Yes \\
\hline Resistance to Man in The Middle attack & Yes & Yes & Yes & Yes \\
\hline USER IMPERSONATINGATTACK & Yes & Yes & Yes & Yes \\
\hline SERVERCOUNTERFEITATTACK & Yes & Yes & Yes & Yes \\
\hline RESISTANCETOFORGERY ATTACK & Yes & Yes & Yes & Yes \\
\hline
\end{tabular}

Table 4. Security aspects comparaison

\subsubsection{The Limitations and weaknesses of CA-KEP [11]}

The CA-KEP approach does not resist to the stolen verifier attack, since it is mandatory to store the fingerprint of the user in order to complete the execution of the protocol. Moreover the transfer of the fingerprint remains problematic.

It is clear that our scheme is more secure than other relevant studies by supporting all security requirements, excluding the RPAKE approach which provides the same security level as our protocol. But we will prove in the next section that our protocol is much more efficient in terms of performance.

\subsection{Implementation Analysis}

Since the CA operations use the simple binary operators that are executed independently for each bit, the CA-based evolution mechanism has an inherent parallelism, and therefore provides an extremely fast and efficient implementation, especially on hardware design. This also prevents denial-of-service (DoS) attacks based on server overload with spurious service requests.

These facts lead to a high speed of authentication of real-time operating performance. Using a conceptual analysis of both the proposed approach and those already in place. The comparative Table 5 shows that the proposed approach takes advantage in terms of cost of communication and calculation.

Note that $\boldsymbol{h}$ denotes for an operation of one-way hash function, $\boldsymbol{r}$ stands for an operation of generation random number, $\boldsymbol{e}$ denotes for an instances of exponential modulo operation, $\boldsymbol{s}$ denotes for an instance of symmetric encryption and " $\boldsymbol{a}$ " denotes for an instance of asymmetric encryption.

\subsubsection{Implementation Limits of CPAKE}

The limits of this scheme are not limited to the security aspect but also in terms of calculation complexity. This schema uses 


\begin{tabular}{|l|l|l|l|l|}
\hline Mutual Authentication Phase & CPAKE[9] & RPAKE[10] & CA-KEP[11] & Proposed \\
\hline Number of message flows & 4 & 2 & 4 & 2 \\
\hline Instances of hash functions (h) & 10 & 6 & 4 & 0 \\
\hline Number of random numbers (r) & 6 & 2 & 2 & 0 \\
\hline Instances of exponential modulo operation (e) & 0 & 0 & 4 & 0 \\
\hline Instances of symmetric encryption (s) & 2 & 0 & 0 & 2 \\
\hline Instances of asymmetric encryption (a) & 0 & 2 & 4 & 2 \\
\hline Intermediate data exchange & $\mathrm{S}_{\mathrm{K}}$ & - & $\mathrm{P}_{\mathrm{K}}, \mathrm{S}_{\mathrm{K}}$ & - \\
\hline Key Confirmation Phase & & & & \\
\hline Number of message flows & 4 & 2 & 2 & 1 \\
\hline Instances of hash functions & - & 6 & 4 & 2 \\
\hline Total & & & & \\
\hline Number of message flows & $\mathbf{8}$ & $\mathbf{4}$ & $\mathbf{6}$ & $\mathbf{3}$ \\
\hline Performance & $\mathbf{1 0 h + 6 r + 2 s}$ & $\mathbf{1 2 h + 2 r + 2 a}$ & $\mathbf{8 h}+\mathbf{2 r}+\mathbf{4 e}+\mathbf{4 a}$ & $\mathbf{2 h} \mathbf{2}+\mathbf{2}+\mathbf{2 a}$ \\
\hline
\end{tabular}

Table 5. Performance Comparison

multiple CA-based hash function instances, which increases the protocol execution time and degrades implementation performance especially when it is about a low-computing devices. In addition this scheme is dependent on intermediate data exchange (a preshared key). The most obvious implementation limit is the number of message flows, 8-way handshake must be done to ensure the authenticated key exchange and key confirmation.

\subsubsection{Implementation Limits of RPAKE}

We can detect gaps in terms of implementation analysis, this schema uses 12 hash function instances, 6 instances for the authentication and key establishment phase and 6 others for the confirmation phase of the key generated. This increases the client-side protocol execution time for a low-capacity device and server-level for a large number of users.

\subsubsection{Implementation Limits of CA-KEP}

This scheme needs 6-way handshake, 8 instances of hash functions and 4 Instances of exponential modulo operation to accomplish authenticated key exchange. Furthermore, the limitations of this approach lie in the fact that the execution of the protocol depends on several intermediate modules independent of the protocol.

The first module is the generator of the secret using the digital fingerprint, it requires complicated processes and costly devices for companies.

Another module required by this protocol is the public key infrastructure module. Each participant must go through an offline certification authority that all other participants trust in order to certify their public keys.

In addition to these limitations, we find an application limit in the case of an application (direct user-to-user authentication) and the prior sharing of the digital fingerprint remains a problem.

\section{Conclusion}

This paper proposes a new cellular automaton based authenticated key exchange scheme that ensure both authentication and session key agreement in a fast and highly secure manner using only a small shared password. The scheme provides a high security and performances level with optimized computational cost, while performed analysis and experiments shows that it resists known attacks. The proposed scheme handle remote user authentication, session key agreement and mutual authentication 
between the user and the server using a simple and elegant computational model. Computational experiments performed to measure the runtime performances shows that the scheme provides best performances with respect to existing CA-based ones. In future works, we plan to extend the scheme to handle situations when multiples $(n>2)$ parties contribute to share a common session cryptographic key.

\section{References}

[1] Diffie, W., Hellman, M. E. (1976). New Directions in Cryptography, In: Proc. 1976 IEEE Intl Symposium on Information Theory, p. 29-40.

[2] Bellovin, S. M., Merritt, M. (1992). Encrypted Key Exchange: Password-Based Protocols Secure Against Dictionary Attacks, In: Proc. 1992 IEEE Intl. Symposium on Research in Security and Privacy, p 72-84.

[3] Gong, L., Lomas, M. L., Needham, R. M., Saltzer, J. H. (1993). Protecting poorly chosen secrets from guessing attacks, IEEE Journal on Selected Areas in Communications, 11 (5) 648-656.

[4] Jablon, D. P. (1997). Strong password-only Authenticated Key Exchange, ACMSIGCOMM Computer Comm Review, p. 5-26.

[5] Boyko, V., MacKenzie, P., Patel, S. (2000). Provably secure password authenticated key exchange using Diffie-Hellman,In: Proc. Eurocrypt, p. 156-171.

[6] MacKenzie, P., Patel, S., Swaminathan, R. (2000). Password authenticated key exchange based on RSA, In: Proc. Asiacrypt, p. $599-613$.

[7] Zhu, F., Wong, D. S., Chen, A. H., Ye, R. (2002). Passwordauthenticated key-exchange based on RSA for imbalanced wirelessnetworks, In: Proc. ISC-2002, p. 150

[8] Bao, F. Security analysis of password authenticated key-exchange protocol, In: Proc. ISC-2003, p 208.

[9] Bhattacharryya, D. K., Nandi, S. (2000). CA based password-only authenticated key exchange. In: Signal Processing Systems. SiPS 2000. 2000 IEEE Workshop on (p. 820-827). IEEE.

[10] Tripathy, S., Nandi, S. (2006). RPAKE: RCA-based Password Authenticated Key Exchange. In: 2006 Annual IEEE India Conference (p. 1-5). IEEE. (September).

[11] Bhuyan, M. H., Bhattacharyya, D. K., Kalita, J. K. (2012). CA-KEP: A Secure CA Based 2-Party Key Exchange Protocol. Journal of Information Assurance and Security, 7 (3) 193-206.

[12] Wolfram, S. (1984). Cellular automata as models of complexity, Naturevol. 311 (419).

[13] Wolfram, S. Cryptography with cellular automata, In: Proc. Advances in Cryptology-Crypto’85, p. 429-432.

[14] Wolfram, S. (1986). Theory and Applications of Cellular Automata, World Scientific, Singapore.

[15] Palchaudhuri, P., Roy Chowdhury, D., Nandi, S., Chattopadhyay, S. (1997). Additive cellular Automata theory and applications, vol.1, IEEE,computer society.

[16] Wolfram, S. (2002). Anew kind of science, Wolfram media Inc. 2002.

[17] Wolfram, S. (1986). Theory and Applications of Cellular Automata, World Scientific, Singapore.

[18] Lind, D., Marcus, B. (1995). An Introduction to Symbolic Dynamics and Coding, Cambridge University Press, Cambridge.

[19] Mora, J. (2005). Procedures for calculating reversible one-dimensionalcellular Automata, Physica.

[20] Kari, J. (1994). Reversibility and surjectivity problems of cellular automata, Journal of Computer and System Sciences, 48 (1) $149-182$.

[21] Kari, J. (1996). Representation of reversible cellular automata with blockpermutations, Math. Syst. Theory, 29 p. 47-61.

[22] Durand-Lose, J. (2001). Representing Reversible Cellular Automata with Reversible Block Cellular Automata, Discrete Mathematics and Theoretical Computer Science ProceedingsAA (DM-CCG), p. 145-154.

[23] Sutner, K. (1995). On the computational complexity of finite Cellular Automata, Journal of Comp and Sys. Sc. 50 (1) 87-97. 
[24] Amoroso, S., Patt, Y. (1972). Decision procedures for Surjectivity andInjectivity of Parallel Maps for Tessellation Structures, Journal of Comput. System Sci. 6, p. 448.

[25] Baghor, S., Faraoun, K. M. (2016). A Novel Fast and Secure Mutual Remote User Authentication Protocol with Session Key Agreement Using Second Order Cellular Automata.

[26] Kari, J. (1992). Cryptosystems based on reversible cellular automata. Manuscript, August.

[27] Choudhury, P. P., Choudhury, D. R., Nandi, S., Chattopadhay, S. (1997). Additive Cellular Automata: Theory and Application, (1) IEEE.

[28] Daemenn., J, Govaerts, R., Vandewalle, J. (1993). A framework for the Design of One-Way Hash Function Based On a Cellular Automaton, LNCS 739, Asiacrypt 91.

[29] Bhattacharyya, D. K. (1997). Efficient One-way Hash Function Design using Cellular Automata, In: Proceedings of the $4^{\text {th }}$ Int'nl Conf. on Advanced Computing, Chennai.

\section{Author Biographies}

Soufiane Baghor is a student at the Djilali Liabes University of Sidi Bel Abbes - Algeria. He is working on authentication schemes, cryptographic protocols and cellular automata. He is currently preparing his PHD thesis on authentication and key exchange protocols using cellular automata.

Kamel Mohamed Faraoun received his master's degree in computer science at the computer science department of DjilaliLiabes University- Sidi-bel-Abbes - Algeria in 2002, his Ph.D degree in computer science, in 2006, and his HDR degree in computer science and intelligent systems, in 2009 From UDL-University. His current research areas include computer security systems; cryptography; genetic algorithms; cellular automata; evolutionary programming and information theory. 